\title{
Introduzione alla conoscenza della sottofamiglia Aleocharinae della Guyana Francese: Parte II ${ }^{1}$ (Coleoptera, Staphylinidae)
}

\author{
Con 41 figure \\ ROBERTO PACE ${ }^{2}$ \\ ${ }^{1} 285^{\circ}$ "Contributo alla conoscenza delle Aleocharinae". \\ ${ }^{2}$ Via Vittorio Veneto, 13, 37032 Monteforte d’Alpone (Verona), Italia. - pace.ent@tiscali.it \\ Published on 2014-07-31
}

\section{Summary}

Description of 11 new species of the tribes Homalotini, Diestotini, Falagriini: Plesiomalota guyminor n. sp., Microchara sulcicollis n. sp., Parasilusa struyvei n. sp., Diestota sensitiva n. sp., Diestota zanettii n. sp., Diestota athetoides n. sp., Gansiella granulosa n. sp., Gansiella guyanensis n. sp., Neolara guyanensis n. sp., Neolara cayennensis n. sp., Tropidera guyanensis n. sp. Additional distributional records are reported for 9 species. Plesiomalota illota (ERICHSON, 1840) is a new combination for Homalota illota ERICHSON, 1840. All new species are illustrated and compared with similar species.

\section{Key words}

Coleoptera, Staphylinidae, Aleocharinae, taxonomy, French Guyana.

\section{Zusammenfassung}

11 neue Arten der Tribus Homalotini, Diestotini, Falagriini werden beschrieben: Plesiomalota guyminor n. sp., Microchara sulcicollis n. sp., Parasilusa struyvei n. sp., Diestota sensitiva n. sp., Diestota zanettii n. sp., Diestota athetoides n. sp., Gansiella granulosa n. sp., Gansiella guyanensis n. sp., Neolara guyanensis n. sp., Neolara cayennensis n. sp., Tropidera guyanensis n. sp. Für 9 Arten werden zusätzliche Daten aufgeführt. Plesiomalota illota (ERICHsON, 1840) ist eine neue Kombination für Homalota illota Erichson, 1840. Jede neue Art wird illustriert und mit ähnlichen Arten verglichen.

\section{Riassunto}

Descrizione di 11 nuove specie delle tribù Homalotini, Diestotini, Falagriini: Plesiomalota guyminor n. sp., Microchara sulcicollis n. sp., Parasilusa struyvei n. sp., Diestota sensitiva n. sp., Diestota zanettii n. sp., Diestota athetoides n. sp., Gansiella granulosa n. sp., Gansiella guyanensis n. sp., Neolara guyanensis n. sp., Neolara cayennensis n. sp., Tropidera guyanensis $\mathrm{n}$. sp. Sono riportati dati distributivi supplementari per 9 specie. Plesiomalota illota (ERICHSON, 1840) è nuova combinazione per Homalota illota ERICHSON, 1840. Ogni specie nuova è illustrata e comparata con specie simili. 


\section{Acronimi}

Gli holotypi e paratypi delle nuove specie e altro materiale sono conservati nei seguenti istituti:

FMNHC: Field Museum of Natural History, Chicago IRSNB: Institut royal des Sciences naturelles de Belgique, Bruxelles

MNHUB: Museum für Naturkunde der HumboldtUniversität, Berlin

SDEI: $\quad$ Senckenberg Deutsches Entomologisches Institut, Müncheberg

\section{HOMALOTINI}

Coenonica puncticollis KraAtz, 1857

Coenonica puncticollis KraAtz, 1857: 46; CAmeron, 1939: 150; PACE, 1984: 15

2 esemplari, Guyane Fr., Chervein, 12.XI.2011, leg. T. Struyve (IRSNB).

Distribuzione: Regione orientale, Madagascar.

\section{DIESTOTINI}

\section{Plesiomalota illota (ERICHSON, 1840) comb. n.}

Homalota illota ERICHson, 1840: 95

1 ㅇ, Guyane Fr., Caussade, 7.XI.2011, leg. T. Struyve (IRSNB).

Distribuzione: Brasile.

Nota: Nuova combinazione stabilita dopo mio esame della serie tipica (MNHUB).

\section{Plesiomalota guyminor $\mathrm{n}$. sp.}

(Figg. 1 e 12-13)

Materiale tipico: Holotype đ`, Guyane Fr., Coralie, 15.XI.2011, leg. T. Struyve (IRSNB).

Paratype: $1 \overbrace{}^{\star}$, stessa provenienza (SDEI).

Descrizione: Lunghezza 1,3 mm. Corpo opaco e bruno, addome giallo-rossiccio con uroterghi liberi quarto e quinto bruni, antenne brune con i due antennomeri basali bruno-rossicci, zampe gialle. Occhi più lunghi della regione postoculare, in visione dorsale. Secondo antennomero più corto del primo, terzo più corto del secondo, quarto a decimo trasversi. Reticolazione di capo e pronoto forte, quella delle elitre confusa, quella dell'addome superficiale. Punteggiatura di capo e pronoto non visibile, quella delle elitre molto fitta ed evanescente. Granulosità dell'addome fine e fitta, ma sugli uroterghi liberi quarto e quinto sparsa. Edeago figg. 12-13.

Comparazioni: L'edeago della nuova specie per lunghezza e forma, in visione ventrale, è simile a quello di $P$. cara PACE, 1996 dell'Ecuador. Se ne distingue per la parte apicale dell'edeago più corta di quella di cara. La punteggiatura del capo di cara è profonda, quella della nuova specie non visibile.

Etimologia: La nuova specie ha nome che significa "la più piccola della Guyana Francese".

\section{Microchara sulcicollis n. sp. (Figg. 2 e 14-16)}

Materiale tipico: Holotype $\sigma^{\star}$, Guyane Fr., Coralie, 16.XI.2011, leg. T. Struyve (SDEI).

Paratype: 1 , stessa provenienza (IRSNB).

Descrizione: Lunghezza 1,3 mm. Corpo lucido e giallo-bruno, elitre brune, antenne brune con i due antennomeri basali bruno-rossicci, zampe giallo-rossicce. Occhi lunghi quanto la regione postoculare, in visione dorsale. Secondo antennomero più corto del primo, terzo più corto del secondo, quarto a decimo trasversi. Reticolazione del capo evidente, ma assente sulla fronte, quella del pronoto forte, quella delle elitre ben visibile, quella dell'addome superficiale. Punteggiatura del capo fitta e poco evidente. Granulosità o punteggiatura del pronoto non visibile. Granulosità delle elitre fitta e superficiale, quella degli uroterghi liberi primo e secondo fitta, quella degli uroterghi liberi terzo a quinto sparsa. Edeago figg. 14-15, spermateca fig. 16.

Comparazioni: Per la forma della spermateca la nuova specie è simile a M. flava PACE, 1983 del Brasile, ma il bulbo basale della spermateca è maggiore nella nuova specie. Il corpo della nuova specie è giallo-bruno con elitre brune, mentre in flava il corpo è giallo-rossiccio. L’edeago della nuova specie ha apice molto stretto per lungo tratto, in visione ventrale, quello di flava per brevissimo tratto.

Etimologia: La nuova specie ha nome che significa "pronoto con solco". 
Parasilusa struyvei n. sp.

(Figg. 3 e 17-20)

Materiale tipico: Holotype $\sigma^{\star}$, Guyane Fr., Caussade, 7.XI.2011, leg. T. Struyve (IRSNB).

Paratype: $1+$, stessa provenienza (SDEI).

Descrizione: Lunghezza 2,2 mm. Corpo lucido e bruno, capo e uroterghi liberi quarto e quinto neri, elitre giallo-rossicce, antenne nero-brune con i tre antennomeri basali giallo-rossicci, zampe giallo-rossicce. Occhi lunghi quanto la regione postoculare, in visione dorsale. Secondo antennomero più corto del primo, terzo più corto del secondo, quarto a decimo trasversi. Reticolazione di capo ed elitre non visibile, quella del pronoto molto superficiale quella dell'addome trasversa e molto evanescente. Punteggiatura di capo e pronoto fitta e superficiale. Granulosità delle elitre fitta ed evidente, quella delladdome saliente e fitta, ma sul quinto urotergo libero molto sparsa. Profonda fossetta mediana trasversa posteriore sul pronoto. Quinto urotergo libero del maschio con due carene laterali tra cui sta posteriormente un saliente granulo. Edeago figg. 17-18, spermateca fig. 19, sesto urotergo libero del maschio fig. 20.

Comparazioni: La nuova specie è simile sia per l'habitus, sia per la forma dell'edeago e della spermateca, oltre che per le parti orali, a P. iheringi Bernhauer, 1908 del Brasile, di cui ho esaminato 5 esemplari della serie tipica (FMNHC). Ledeago della nuova specie non è ampiamente arcuato al lato ventrale come in iheringi, ma strettamente arcuato. Il sesto urotergo libero del maschio della nuova specie ha due spine e due lobi al margine posteriore, quello di iheringi è ondulato tra due corte spine laterali.

Etimologia: La nuova specie è dedicata al suo raccoglitore Tim Struyve, entomologo e consulente di pianificazione di Mechelen, Fiandre, Belgio.

\section{Diestota (Apheloglossa) ecuadorensis PACE, 1996}

Diestota (Apheloglossa) ecuadorensis PACE, 1996: 431

9 esemplari, Guyane Fr., Caussade, 7.XI.2011, leg. T. Struyve (IRSNB, SDEI).

Distribuzione: Ecuador.

\section{Diestota (Apheloglossa) loretensis PACE, 2008}

Diestota loretensis PACE, 2008: 271

$20^{\star} o^{\star}$, Guyane Fr., Caussade, 7.XI.2011, leg. T. Struyve (IRSNB, SDEI).

Distribuzione: Perù.
Diestota (Apheloglossa) sensitiva n. sp.

(Figg. 4 e 21-22)

Materiale tipico: Holotype $\sigma^{*}$, Guyane Fr., Charvein, 12.XI.2011, leg. T. Struyve (IRSNB).

Descrizione: Lunghezza 1,8 mm. Corpo lucido. Capo nero, pronoto rossiccio, elitre brune, addome giallo-rossiccio con quarto urotergo libero bruno, antenne brune con i tre antennomeri bruno-rossicci, zampe giallo-rossicce. Occhi più lunghi della regione postoculare, in visione dorsale. Secondo antennomero più corto del primo, terzo più corto del secondo, quarto a decimo trasversi. Reticolazione di capo e pronoto evidente, quella di elitre e addome superficiale, sul quarto urotergo libero trasversa, sul quinto assente. Punteggiatura del capo invisibile, quella del pronoto molto fitta e fine. Granulosità di elitre e addome fine, fitta e saliente. Appiattimento mediano posteriore del pronoto. Edeago figg. 21-22.

Comparazioni: Habitus, colore del corpo e forma dell'edeago della nuova specie sono simili a quelli di D. galapagosensis (PACE, 1985) delle isole Galapagos. La nuova specie se ne distingue per la base dell'addome giallo-rossiccio e non bruno-rossiccio come in galapagosensis, e per l'apice dell'edeago in visione ventrale, largo e non molto stretto come in galapagosensis.

Etimologia: La nuova specie prende nome dalla presenza di numerosi pori sensitivi della parte apicale dell'edeago.

\section{Diestota (Apheloglossa) zanettii n. sp.}

(Figg. 5 e 23-25)

Materiale tipico: Holotype $\sigma^{\star}$, Guyane Fr., Caussade, 7.XI.2011, leg. T. Struyve (SDEI).

Descrizione: Lunghezza 1,8 mm. Corpo lucido e nerobruno, elitre brune, antenne brune con i tre antennomeri basali bruno-rossicci, zampe giallo-rossicce. Occhi lunghi quanto la regione postoculare, in visione dorsale. Secondo antennomero lungo quanto il primo, terzo più corto del secondo, quarto a decimo trasversi. Reticolazione dell'avancorpo evidente, quella dell'addome trasversa e ben visibile. Granulosità del corpo fitta e distinta, quella del quinto urotergo libero del maschio sparsa. Impressione mediana posteriore del pronoto trasversa. Edeago figg. 23-24, sesto urotergo libero del maschio fig. 25.

Comparazioni: Il sesto urotergo libero del maschio della nuova specie è simile a quello di D. ecuadorensis PACE, 1996 dell'Ecuador, ma i lobi tra le spine laterali del margine posteriore del sesto urotergo libero del maschio sono sei, mentre quelli di ecuadorensis sono quattro. Ledeago della nuova specie è largamente arcuato al lato ventrale, quello di ecuadorensis strettamente arcuato. 
L'apice dell'edeago della nuova specie è stretto in visione ventrale, largo in ecuadorensis.

Etimologia: La nuova specie è dedicata allo studioso di Staphylinidae Prof. Adriano Zanetti di Verona, che ha contribuito a farmi pervenire gli esemplari di Aleocharinae della Guyana Francese raccolti da T. Struyve .

\section{Diestota (Apheloglossa) athetoides n. sp.}

(Figg. 6 e 26-28)

Materiale tipico: Holotype $\sigma^{\star}$, Guyane Fr., Saramaka, 13.XI.2011, leg. T. Struyve (SDEI).

Paratypes: 23 esemplari, stessa provenienza (IRSNB, SDEI).

Descrizione: Lunghezza $1,7 \mathrm{~mm}$. Corpo lucido, capo bruno, pronoto ed elitre giallo-bruni, addome giallo-rossiccio con quarto urotergo libero bruno, antenne brune con i tre antennomeri basali giallo-rossicci, zampe giallo-rossicce. Occhi lunghi quanto la regione postoculare, in visione dorsale. Secondo antennomero più corto del primo, terzo più corto del secondo, quarto a decimo trasversi. Reticolazione di capo ed elitre evidente, quella del pronoto forte, quella dell'addome superficiale. Punteggiatura del capo molto fitta ed evidente. Granulosità del pronoto non visibile, quella di elitre e addome superficiale. Sul pronoto due deboli solchi mediani. Edeago figg. 26-27, spermateca fig. 28.

Comparazioni: L'edeago molto arcuato della nuova specie è simile a quello di $D$. calida Bernhauer, 1920 del Perù di cui ho esaminato l'olotipo maschio (FMNHC). La nuova specie se ne distingue per l'edeago ancor più arcuato, con apice largo, in visione ventrale, mentre in calida è stretto. Il sesto urotergo libero del maschio della nuova specie ha il margine posteriore lineare, quello di calida è lobato.

Etimologia: La nuova specie ha nome che significa "con aspetto di Atheta".

\section{Eudera bisulcata (ERICHSON, 1840)}

Homalota bisulcata ERICHSON, 1840: 117

Ophioglossa bisulcata: Bernhauer \& Scheerpeltz, 1926: 569

Eudera bisulcata: PACE, 1996: 397

3 esemplari, Guyane Fr., Caussade, 7.XI.2011, leg. T. Struyve; $1 \sigma^{\star}$, Guyane Fr., Coralie, 16.XI.2011, leg. T. Struyve (IRSNB, SDEI).

Distribuzione: Colombia e Brasile.

Nota: Serie tipica da me esaminata composta di 1 $\mathrm{n}^{\circ} 5454$ (MNHUB).

\section{Eudera didyma (ERICHSON, 1840)}

Homalota didyma ERICHSON, 1840: 116

Ophioglossa didyma: Bernhauer \& SCHEERPELtz, 1926: 570

Eudera didyma: PACE, 1996: 397

$10^{\star}$, Guyane Fr., Barrage Petit Saut, 10.XI.2011, leg. T. Struyve (IRSNB).

Distribuzione: Brasile, Venezuela, Colombia, Guadalupa e S. Thomas.

Nota: Serie tipica da me esaminata composta di $3 \sigma^{\top} o^{\top}$ e 2 우 우 $\mathrm{n}^{\circ} 5453$ (MNHUB).

\section{Gansiella peruviana PACE, 2008}

Gansiella peruviana PACE, 2008: 275

10 esemplari, Guyane Fr., Caussade, 7.XI.2011, leg. T. Struyve; 6 esemplari, Guyane Fr., Tonate, 6.XI.2011, leg. T. Struyve (IRSNB, SDEI).

Distribuzione: Perù.

Gansiella granulosa n. sp.

(Figg. 7 e 29-31)

Materiale tipico: Holotype $\sigma^{*}$, Guyane Fr., Caussade, 7.XI.2011, leg. T. Struyve (IRSNB).

Paratype: 1 , stessa provenienza (SDEI).

Descrizione: Lunghezza 2,1 mm. Avancorpo debolmente lucido, addome lucido. Corpo rossiccio, base delle elitre giallo-rossiccia, antenne bruno-rossicce con i tre antennomeri basali e l'undicesimo giallo-rossicci, zampe giallo-rossicce. Occhi più corti della regione postoculare, in visione dorsale. Secondo antennomero più corto del primo, terzo più lungo del secondo, quarto e quinto più lunghi che larghi, sesto lungo quanto largo, settimo a decimo trasversi. Reticolazione del capo evidente, quella di pronoto ed elitre molto superficiale, quella dell'addome invisibile. Punteggiatura del capo molto fitta ed evidente. Granulosità di pronoto ed elitre fine e fitta, ben visibile, quella dell'addome saliente, ma sul terzo urotergo libero composta di granuli lunghi sulla metà posteriore e da una fila trasversa basale di granuli. Granulosità del quarto urotergo libero composta di granuli longitudinali salienti, quelli del quinto meno salienti. Pronoto con appiattimento mediano posteriore della sua superficie. Quarto urotergo libero con carene basali corte. Edeago figg. 29-30, spermateca fig. 31 .

Comparazioni: La nuova specie è distinta da quelle note per l'avancorpo fittamente granuloso e quasi opaco (avan- 
corpo sparsamente granuloso e lucido nelle altre specie note), e per l'edeago meno lungo di quello delle specie già descritte.

Etimologia: La nuova specie prende nome dalla fitta granulosità del suo corpo.

\section{Gansiella guyanensis n. sp.}

(Figg. 8 e 32-34)

Materiale tipico: Holotype $\sigma^{\star}$, Guyane Fr., Tonate, 6.XI.2011, leg. T. Struyve (SDEI).

Paratypes: 5 esemplari, stessa provenienza (IRSNB, SDEI).

Descrizione: Lunghezza 3,1 mm. Corpo lucido e giallo-rossiccio, angoli posteriori esterni delle elitre e quarto urotergo libero bruni, antenne rossicce con i tre antennomeri basali e il decimo e l'undicesimo giallo-rossicci, zampe giallo-rossicce. Occhi più corti della regione postoculare, in visione dorsale. Secondo antennomero più corto del primo, terzo più lungo del secondo, quarto a sesto più lunghi che larghi, settimo e ottavo lunghi quanto larghi, nono e decimo trasversi. Reticolazione del corpo non visibile. Punteggiatura del capo fine e fitta. Granulosità di pronoto ed elitre fine e fitta. Alcuni punti sparsi sull'addome. Pronoto con larga e profonda depressione mediana posteriore. Edeago figg. 32-33, spermateca fig. 34 .

Comparazioni: La nuova specie è simile a G. peruviana PACE, 2008 del Perù e Guyana Francese. È distinta da essa e da granulosa per i caratteri dati nella seguente chiave:

Etimologia: La nuova specie è dedicata alla Guyana Francese.

1. Quarto urotergo libero con carene basali, pronoto fortemente granuloso e poco ristretto all'indietro, edeago lungo 0,22 $\mathrm{mm}$. Corpo lungo 2,1 $\mathrm{mm}$

G. granulosa n. sp.

Quarto urotergo libero senza carene basali, pronoto finemente granuloso e fortemente ristretto all'indietro, edeago lungo $0,3-0,41$

2

2. Undicesimo antennomero giallo-rossiccio, decimo bruno, pronoto debolmente impresso sulla metà posteriore, edeago debolmente arcuato, lungo 0,3 $\mathrm{mm}$. Corpo lungo 2,8 $\mathrm{mm}$ G. peruviana PACE

Antennomeri undicesimo e decimo giallo-rossicci, pronoto largamente e profondamente impresso sulla metà posteriore, edeago fortemente arcuato e lungo 0,41 mm. Corpo lungo 3,1 mm

G. guyanensis n. sp.

\section{FALAGRIINI}

Falagria (Myrmecocephalus) concinna (ERICHSON, 1840)

Falagria concinna ERICHSON, 1840: 51

Myrmecocephalus concinnus: NAKANE \& al., 1963: 98

1 ㅇ, Guyane Fr., Coralie, 15.XI.2011, leg. T. Struyve (IRSNB).

Distribuzione: Specie cosmopolita.

Nota: Da me esaminato il lectotypus of $\mathrm{n}^{\circ} 5288$ del Brasile (MNHUB). Da altri autori questa specie è citata come Myrmecocephalus concinnus.

\section{Meronera deliciata (ERICHSON, 1840)}

Falagria deliciata ERICHSON, 1840: 56

Meronera deliciata: BeRNHAUER \& SCHEERPELTZ, 1926: 580

1 ðै, Guyane Fr., Caussade, 7.XI.2011, leg. T. Struyve; 3 esemplari, Guyane Fr., Barrage Petit Saut, 10.XI.2011, leg. T. Struyve (IRSNB, SDEI).

Distribuzione: Brasile.

Nota: Da me esaminato l'olotipo 우, $\mathrm{n}^{\circ} 5302$ (MNHUB).

\section{Neolara guyanensis n. sp.}

(Figg. 9 e 35-37)

Materiale tipico: Holotype + , Guyane Fr., Caussade, 6.XI.2011, leg. T. Struyve (SDEI).

Paratypes: 3 우, stessa provenienza; 11 esemplari, Guyane Fr., Tonate, 6.XI.2011, leg. T. Struyve; 6 esemplari, Guyane Fr., Coralie, 15.XI.2011, leg. T. Struyve; $10^{\text {*ै, }}$ Guyane Fr., Coralie, 16.XI.2011, leg. T. Struyve (IRSNB, SDEI).

Descrizione: Lunghezza 2,3 mm. Corpo lucido e nero-bruno, uroterghi liberi basali primo e secondo bruno-rossicci, antenne nere con i due antennomeri basali bruno-rossicci, zampe brune, tarsi rossicci. Occhi più corti della regione postoculare, in visione dorsale. Secondo antennomero più corto del primo, terzo lungo quanto il secondo, quarto e quinto più lunghi che larghi, sesto lungo quanto largo, settimo e decimo trasversi. Reticolazione del capo molto superficiale, quella di pronoto ed elitre evidente, quella dell'addome non visibile. Punteggiatura del capo fitta ed evidente, assente tra le antenne, quella dell'addome molto sparsa. Granulosità di pronoto ed elitre fitta e saliente. Pronoto con una profonda fossetta mediana posteriore. Edeago figg. 35-36, spermateca fig. 37. 
Comparazioni: Per la corta parte prossimale della spermateca, la nuova specie è distinta da quelle note che hanno la parte prossimale della spermateca ampiamente arcuata o descrivente una spira. La profonda fossetta mediana posteriore del pronoto non è presente nelle altre specie note del genere.

Etimologia: La nuova specie è dedicata alla Guyana Francese.

\section{Neolara cayennensis n. sp.}

(Figg. 10 e 38-39)

Materiale tipico: Holotype ${ }^{\star}$, Guyane Fr., Barrage Petit Saut, 10.XI.2011, leg. T. Struyve (IRSNB).

Descrizione: Lunghezza $1,8 \mathrm{~mm}$. Corpo lucido e giallo-rossiccio, addome nero, uroterghi liberi primo e secondo giallo pallido con base e fascia mediana brune, antenne giallo-rossicce con antennomeri settimo a undicesimo bruni, zampe gialle con tibie brune e femori posteriori bruni con le estremità giallo-rossicce. Occhi lunghi quanto la regione postoculare, in visione dorsale. Secondo antennomero più corto del primo, terzo lungo quanto il secondo, quarto a sesto più lunghi che larghi, settimo e ottavo lunghi quanto larghi, nono e decimo trasversi. Reticolazione del capo evidente, quella del resto del corpo non visibile. Punteggiatura del capo quasi invisibile, quella di pronoto ed elitre fitta e superficiale. Granulosità dell'addome ridotta alla presenza di qualche granulo. Edeago figg. 38-39.

Comparazioni: L'edeago e il colore della base dell'addome indicano l'affinità della nuova specie con N. alboguttata (ERICHSON, 1840) di cui ho esaminato l'olotipo ㅇ $\mathrm{n}^{\circ} 5803$ della Colombia (MNHUB) e vari $0^{\top} o^{\star}$ associati a 우 우 del Brasile (MNHUB). Il penultimo antennomero è trasverso nella nuova specie, più lungo che largo in alboguttata. La macchia omerale gialla e quella degli angoli posteriori esterni di alboguttata mancano nella nuova specie. L'edeago della nuova specie è lungo $0,2 \mathrm{~mm}$, quello di alboguttata $0,32 \mathrm{~mm}$.

Etimologia: La nuova specie è dedicata alla città di Cayenne, capitale della Guyana Francese.

\section{Tropidera guyanensis n. sp. \\ (Figg. 11 e 40-41)}

Materiale tipico: Holotype $\sigma^{x}$, Guyane Fr., Caussade, 6.XI.2011, leg. T. Struyve (SDEI).

Descrizione: Lunghezza 3,4 mm. Specie attera. Corpo lucido e rossiccio, antenne rossicce con gli antennomeri basali primo a sesto giallo-rossicci, zampe rossicce con tibie e tarsi giallo-rossicci. Occhi lunghi quanto la regione postoculare, in visione dorsale. Secondo antennomero più corto del primo, terzo più lungo del secondo, quarto a settimo più lunghi che larghi, ottavo a decimo lunghi quanto larghi. Reticolazione del corpo invisibile. Punteggiatura di capo e pronoto fine e superficiale. Granulosità delle elitre poco fitta ed evanescente, quella dell'addome rappresentata da qualche granulo. Edeago figg. 40-41.

Comparazioni: Per l'habitus e la forma dell'edeago, la nuova specie è simile a T. jenseni Bernhauer, 1908 dell'Argentina, di cui ho esaminato la serie tipica di $10^{*}$ e 1 ㅇ (FMNHC). La nuova specie se ne distingue per avere l'edeago molto ristretto nella regione intermedia, in visione ventrale. In jenseni è lievemente ristretto. In visione ventrale, l'apice dell'edeago della nuova specie è strettamente arcuato, mentre lo è largamente in jenseni.

Etimologia: La nuova specie è dedicata alla Guyana Francese. 


\section{Ringraziamenti}

Per avermi affidato in studio il materiale oggetto del presente lavoro rivolgo i miei più cordiali ringraziamenti al collega e naturalista belga Tim Struyve di Mechelen. Per il prestito di tipi ringrazio il Dr. A. Newton del Field Museum of Natural History di Chicago e il Dr. Manfred Uhlig del Museum für Naturkunde der Humboldt-Universität, Berlino.

\section{Bibliografia}

Bernhauer, M. 1908: Beitrag zur Staphylinidenfauna von Südamerika. - Archiv für Naturgeschichte 74: 283-372.

Bernhauer, M. 1920: Neue Aleocharini aus Südamerika. - Archiv für Naturgeschichte 86: 141-170.

Bernhauer, M. \& Scheerpeltz, O. 1926: Coleopterorum Catalogus, pars 82, Staphylinidae VI: 499-988, Berlin.

CAmeron, M. 1939: The Fauna of British India, including Ceylon and Burma. Coleoptera, Staphylinidae. Vol. IV: 410 pp., London.

Erichson, W. F. 1840: Genera et Species Staphylinorum Insectorum Coleopterorum Familiae. - Berlin: 954 pp.

KraAtz, G. 1857: Beiträge zur Kenntnis der Termitophilen. - Linnaea Entomologica 11: 44-56.
Nakane, T.; Ohbayashi, R.; Nomura, S. \& Kurosawa, Y. 1963: Iconographia Insectorum Japonicorum, Colore Naturali Edith. - Vol. 2 (Coleoptera): 443 pp.

PACE, R. 1983: Nuove Aleocharinae Brasiliane e boliviane del Museo Zoologico Dell'Università Humboldt di Berlino (Coleoptera Staphylinidae) (XXXVIII tris Contributo alla conoscenza delle Aleocharinae). Giornale italiano d'Entomologia 2: 295-316, 92 figg.

PACE, R. 1984: Aleocharinae delle Mascarene, parte I: Tribù Myllaenini, Pronomaeini, Oligotini e Bolitocharini (Coleoptera, Staphylinidae) (XLV Contributo alla conoscenza delle Aleocharinae). Revue suisse de Zooogie 91: 3-36.

PACE, R. 1985: Aleocharinae delle Isole Galapagos (Coleoptera, Staphylinidae) (LXXIII Contributo alla conoscenza delle Aleocharinae). - Annales de la Société entomologique de France 22: 449-456.

Pace, R. 1996: Aleocharinae della Colombia e dell'Ecuador: Parte I (Coleoptera, Staphylinidae). Revue suisse de Zoologie 103: 395-437.

PACE, R. 2008: New records of Aleocharinae from Ecuador and Peru, with the description of new species, new subgenera and new genera (Coleoptera, Staphylinidae): pp. 225-398. - In: Giachino P. M. (ed.) 2008: Biodiversity of South America I. - Memoirs on Biodiversity, World Biodiversity Association onlus, Verona 1: 496 pp. 


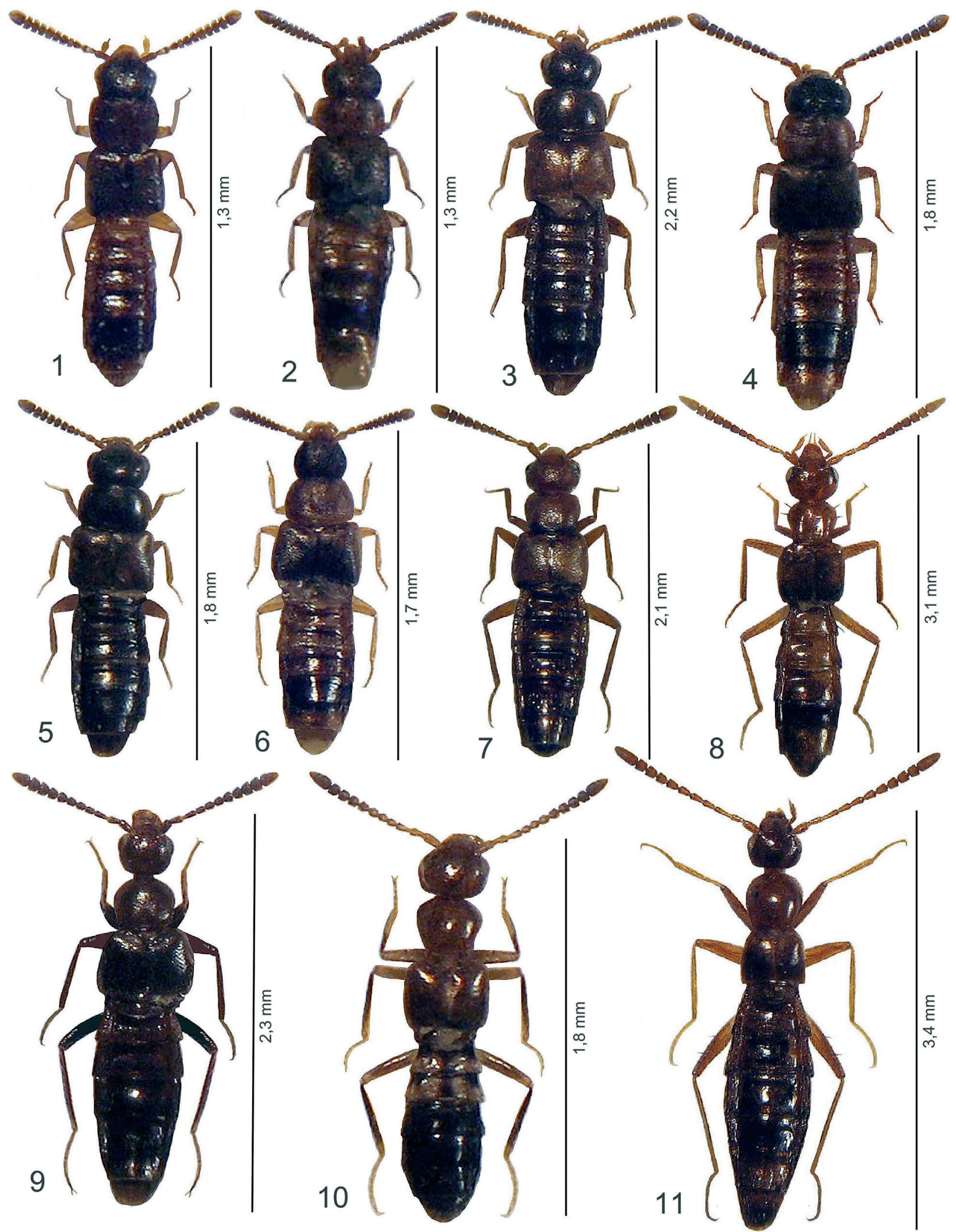

Figg. 1-12: Habitus. 1. Plesiomalota guyminor n. sp.; 2. Microchara sulcicollis n. sp.; 3. Parasilusa struyvei n. sp.; 4. Diestota (Apheloglossa) sensitiva n. sp.; 5. Diestota (Apheloglossa) zanettii n. sp.; 6. Diestota (Apheloglossa) athetoides n. sp.; 7. Gansiella granulosa n. sp.; 8. Gansiella guyanensis n. sp.; 9. Neolara guyanensis n. sp.; 10. Neolara cayennensis n. sp.; 11. Tropidera guyanensis $\mathrm{n}$. $\mathrm{sp}$. 

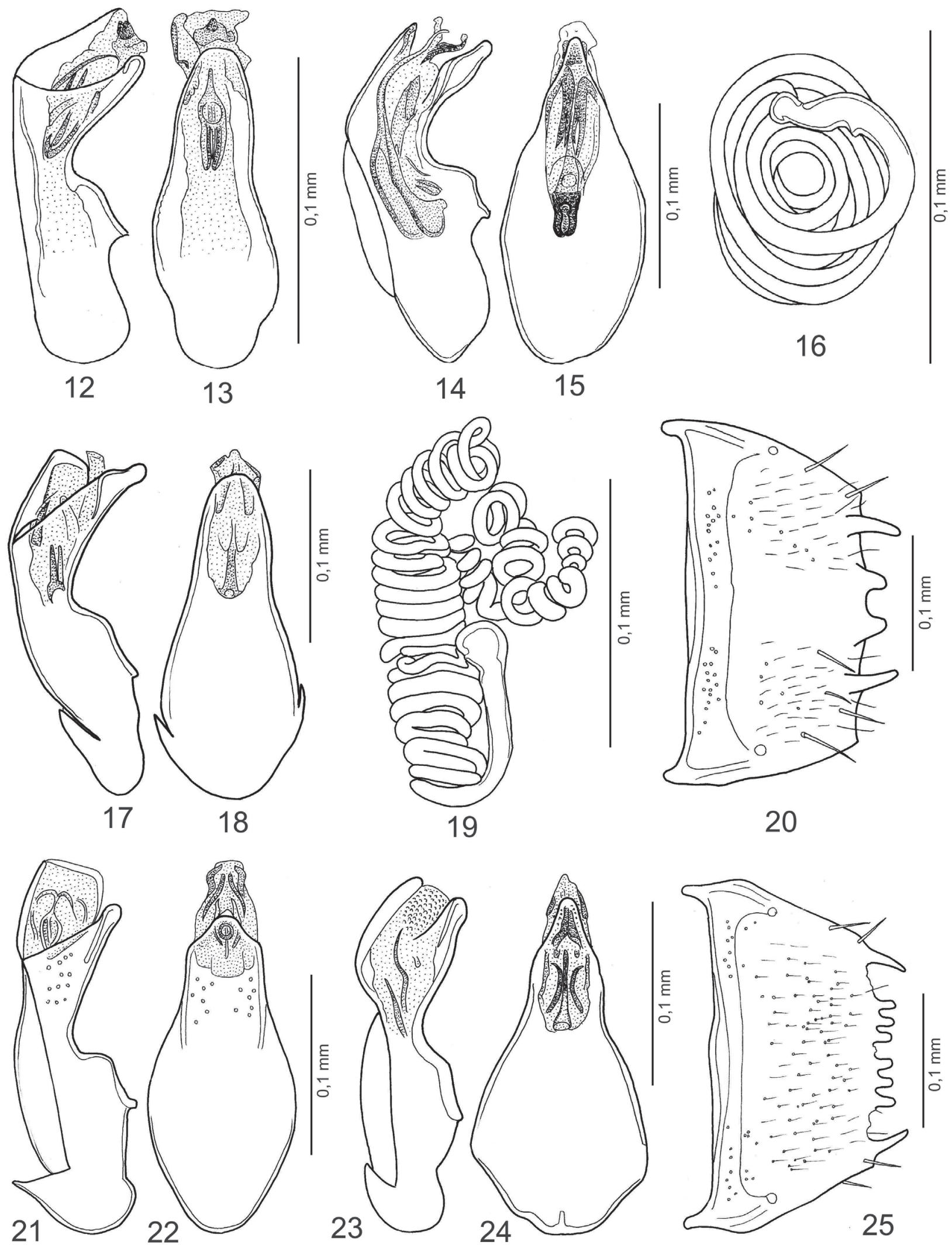

Figg. 12-25: Edeago in visione laterale e ventrale, spermateca e sesto urotergo libero del maschio. 12-13. Plesiomalota guyminor n. sp.; 14-16. Microchara sulcicollis n. sp.; 17-20. Parasilusa struyvei n. sp.; 21-22. Diestota (Apheloglossa) sensitiva n. sp.; 23-25. Diestota (Apheloglossa) zanettii n. sp. 

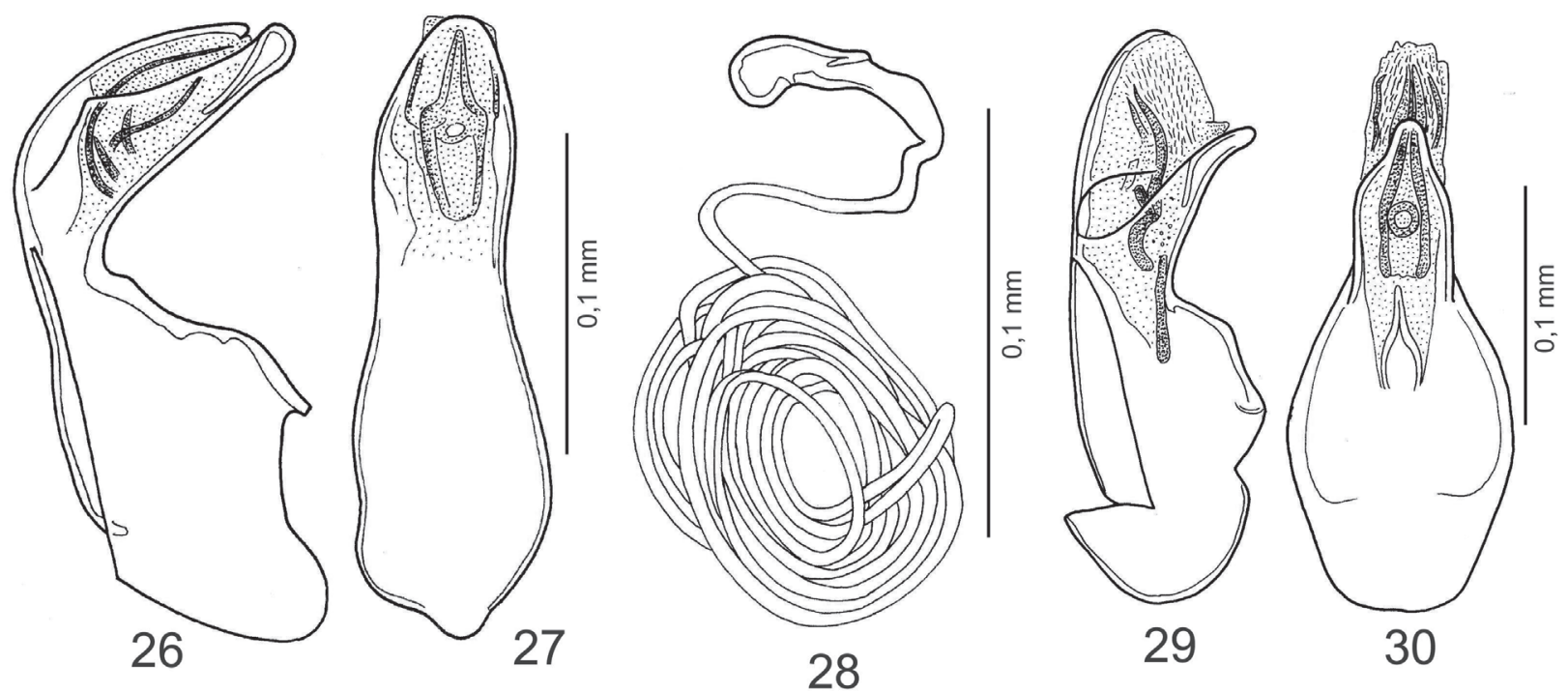

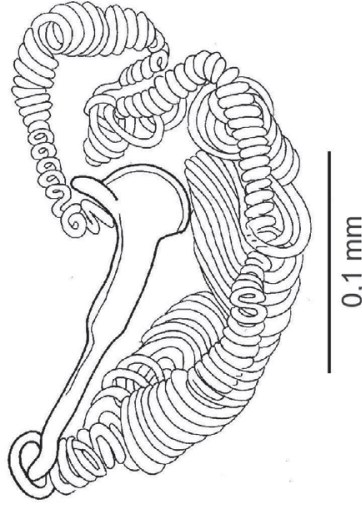

31
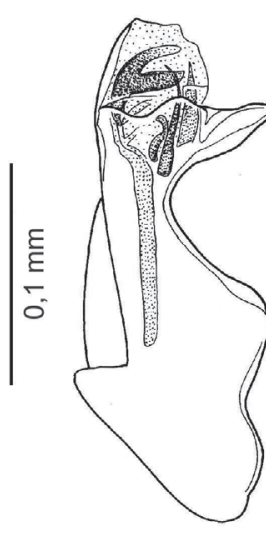

32

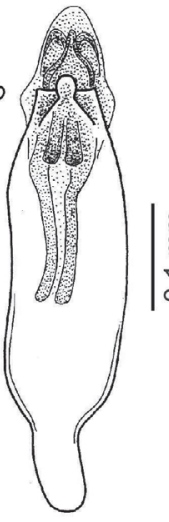

33

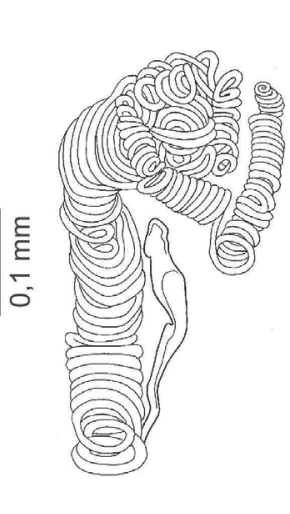

34
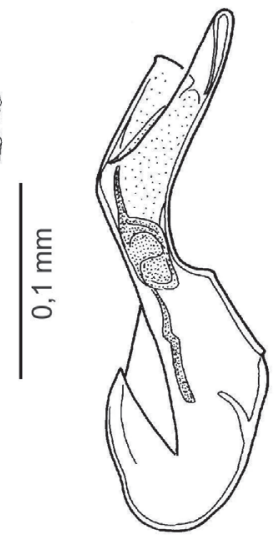

35

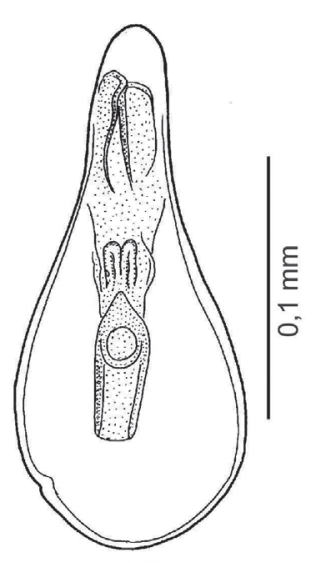

36
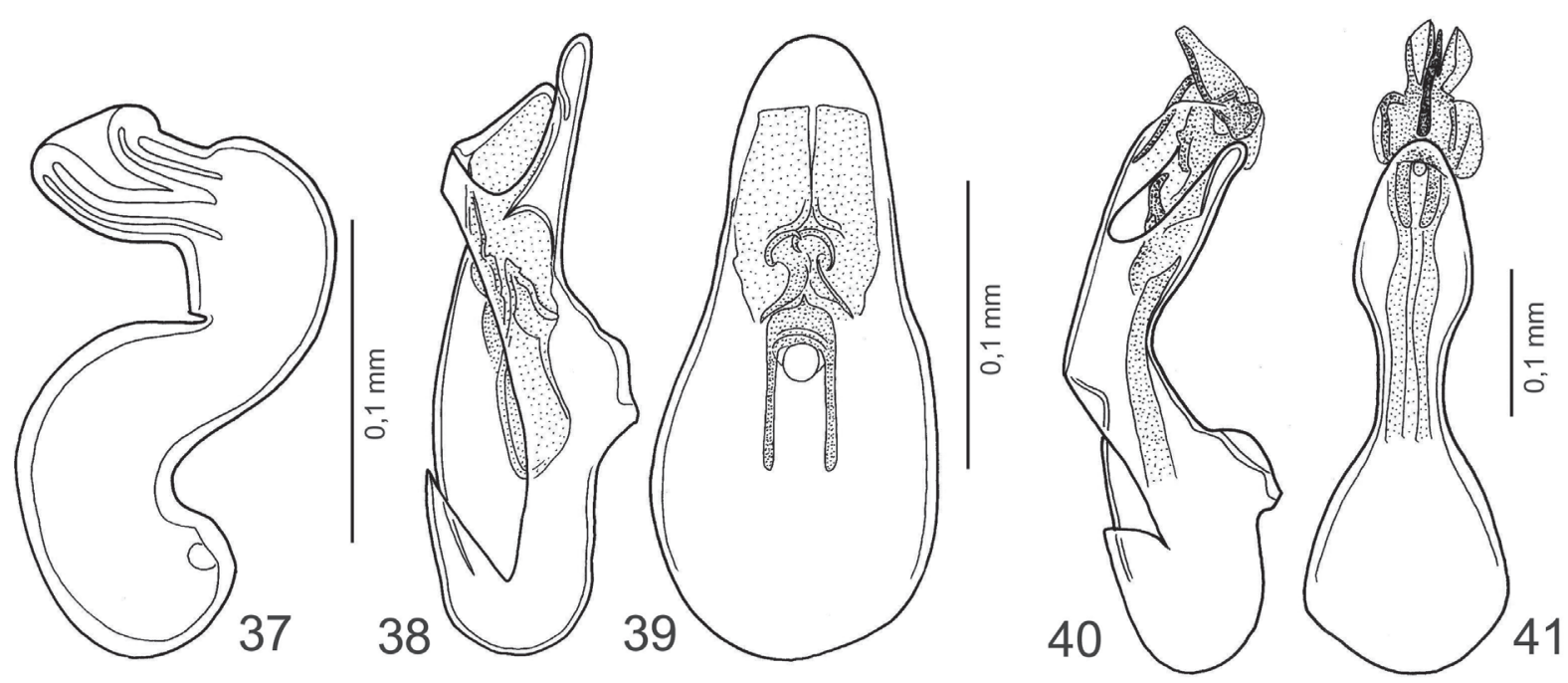

Figg. 26-41: Edeago in visione laterale e ventrale e spermateca. 26-28. Diestota (Apheloglossa) athetoides n. sp.; 29-31. Gansiella granulosa n. sp.; 32-34. Gansiella guyanensis n. sp.; 35-37. Neolara guyanensis n. sp.; 38-39. Neolara cayennensis $\mathrm{n}$. sp.; 40-41. Tropidera guyanensis $\mathrm{n}$. sp. 